\title{
Emerging Roles of Downstream of Kinase 3 in Cell Signaling
}

\author{
Jia Tong Loh ${ }^{1,2 *}$, Joey Kay Hui Teo ${ }^{1,2}$, Hong-Hwa Lim ${ }^{1,3}$ and Kong-Peng Lam ${ }^{1,2,4,5 *}$ \\ ${ }^{1}$ Bioprocessing Technology Institute, Agency for Science, Technology and Research, Singapore, Singapore, ${ }^{2}$ Singapore \\ Immunology Network, Agency for Science, Technology and Research, Singapore, Singapore, ${ }^{3}$ Institute of Molecular and \\ Cell Biology, Agency for Science, Technology and Research, Singapore, Singapore, ${ }^{4}$ Department of Microbiology and \\ Immunology, Yong Loo Lin School of Medicine, National University of Singapore, Singapore, Singapore, ${ }^{5}$ School of \\ Biological Sciences, College of Science, Nanyang Technological University, Singapore, Singapore
}

\section{OPEN ACCESS}

Edited by:

Uday Kishore,

Brunel University London, United Kingdom

Reviewed by:

Laura Eugenia Velazquez, Institut National de la Santé et de la

Recherche Médicale (INSERM),

France

Sunil Kumar Verma,

Centre for Cellular \& Molecular

Biology (CCMB), India

*Correspondence: Jia Tong Loh

loh_jia_tong@bti.a-star.edu.sg Kong-Peng Lam lam_kong_peng

@immunol.a-star.edu.sg

Specialty section:

This article was submitted to

Molecular Innate Immunity,

a section of the journal

Frontiers in Immunology

Received: 27 May 2020

Accepted: 10 September 2020

Published: 29 September 2020

Citation:

Loh JT, Teo JKH, Lim H-H and Lam K-P (2020) Emerging Roles of Downstream of Kinase 3 in Cell

Signaling.

Front. Immunol. 11:566192. doi: 10.3389/fimmu.2020.566192
Downstream of kinase (Dok) 3 is a member of the Dok family of adaptor proteins known to regulate signaling pathways downstream of various immunoreceptors. As Dok-3 lacks intrinsic catalytic activity, it functions primarily as a molecular scaffold to facilitate the nucleation of protein complexes in a regulated manner and hence, achieve specificity in directing signaling cascades. Since its discovery, considerable progress has been made toward defining the role of Dok-3 in limiting B cell-receptor signaling. Nonetheless, Dok-3 has since been implicated in the signaling of Toll-like and C-type lectin receptors. Emerging data further demonstrate that Dok-3 can act both as an activator and inhibitor, in lymphoid and non-lymphoid cell types, suggesting Dok-3 involvement in a plethora of signal transduction pathways. In this review, we will focus on the structure and expression profile of Dok-3 and highlight its role during signal transduction in B cells, innate cells as well as in bone and lung tissues.

Keywords: Dok-3, adaptor, cell signaling, B cells, innate cells

\section{INTRODUCTION}

The specific response of cells to environmental stimuli requires crosstalk between multiple signaling pathways, and such specificity in signaling is achieved through a class of proteins known as adaptors, which link specific protein partners together, in a reversible manner, via their protein binding modules to elicit the appropriate cellular response (1). Downstream of kinase (Dok) is a family of adaptor proteins comprising of seven structurally related members, Dok-1 to -7 . Downstream of kinase proteins do not possess any intrinsic catalytic activity, and they function as molecular scaffold to facilitate protein-protein interaction through their distinct protein-binding domains. As their names imply, they play a role downstream of protein tyrosine kinases (PTKs) by connecting these kinases with their downstream effectors, thereby adapting them to various signaling cascades crucial to cellular functions in a spatially and temporally regulated way (2).

Of the seven members in the Dok adaptor family, Dok-1, -2 and -3 are found mainly in hematopoietic cells $(3,4)$ while the rest are found in other tissues $(5,6)$. In this review, we will focus on one member of the Dok family adaptor - Dok-3, which was co-discovered by Cong and colleagues as a protein that bound the cytoplasmic PTK Abl (7), and Lemay and colleagues as a heavily tyrosine phosphorylated protein that was associated with the Src homology 2 
domain-containing inositol $5^{\prime}$-phosphatase (SHIP) and PTK, Csk (8), and discuss how it functions as a molecular integrator of various immune as well as non-immune signaling pathways.

\section{STRUCTURE OF Dok-3}

Downstream of kinase 3 is a relatively small protein comprising of approximately 496 amino acids. At the amino terminus, Dok3 contains a pleckstrin homology $(\mathrm{PH})$ domain involved in plasma membrane localization via its interaction with membrane phospholipids. Most of the PTKs which phosphorylate Dok3 , as well as its downstream binding partners, are localized to cellular membranes; hence the $\mathrm{PH}$ domain is crucial to the membrane localization of Dok-3 for the induction of its function. The $\mathrm{PH}$ domain is followed by a central phosphotyrosine binding (PTB) domain which facilitates the binding of Dok-3 to phosphotyrosine-containing consensus motif NPXpY found in partnering proteins. This enables Dok-3 interaction with its upstream PTKs, and the initiation of its function as a scaffold protein to nucleate the formation of multimeric protein complexes. At the carboxyl terminus, there is a proline-rich region with multiple tyrosine residues which can serve as docking sites for SH2-containing proteins upon phosphorylation $(2,7$, 8) (Figure 1).

Even though the seven Dok family members share structural similarities characterized by an amino-terminal $\mathrm{PH}$ domain followed by a central PTB domain, phylogenetic analysis revealed that they cluster into three different subgroups consisting of Dok-1/2/3, Dok-4/5/6, and Dok-7 respectively (2) (Figure 2). Within the subgroup, the PH and PTB domains of Dok-3 share extensive sequence homology with that of Dok-1 and -2. In particular, a Dok homology (DKH) sequence motif (WPxxxLRxxGxDxxxFxFExGR) has been defined in their PTB domains based on the high sequence similarity observed (9). On the other hand, no conservation in sequence is observed in their carboxy-terminal proline-rich region, possibly accounting for the diverse physiological roles and specificities of Dok-1, -2 and -3 toward different signal-transducing molecules.

\section{EXPRESSION OF Dok-3}

Unlike Dok-4, -5 , and -6 which are preferentially expressed in neural cells and Dok-7 in skeletal muscles $(5,6)$, Dok-1, -2, and -3 are found mainly in hematopoietic cells and known to be involved in a variety of immunoreceptor signaling pathways $(3,4)$. Downstream of kinase 3 is abundantly expressed in lymphoid organs such as spleen and bone marrow, but not in the thymus. Correspondingly, high levels of Dok-3 were found in B cells, macrophages, and neutrophils, while undetectable or non-uniform expression of Dok-3 was observed in $\mathrm{T}$ cells and their relevant cell lines. Among non-lymphoid tissues, Dok-3 was found predominantly in lungs, moderate levels were detected in the appendix, small intestine, colon, reproductive organs, and urinary bladder, while very low levels were found in other organs like liver, kidney, and brain $(7,8,10)$. We will further elaborate on the known physiological role of Dok-3 in each cell type or tissue in the following sections.

\section{Dok-3 SIGNALING}

Downstream of kinase 3 has been demonstrated to participate in the signaling of B cell-receptor (BCR) in B cells $(7,8,11-$ 16), and Toll-like receptors (TLR) and C-type lectin receptors (CLR) in innate immune cells such as macrophages (17-20) and neutrophils (21). Since Dok-3 is an adaptor, it has been shown to bind a variety of molecules involved in intracellular signal transduction (Figure 1). Examples of these molecules involved PTKs such as Abl (7), Lyn (14), and Csk (8), serine/threonine kinase TANK-binding kinase (TBK) 1 (20), lipid phosphatase SHIP-1 $(8,12,13)$, protein serine/threonine phosphatase 1 (PP1) (21), E3 ubiquitin ligase $\mathrm{Cbl}(15,18)$, and also, other adaptor molecules such as growth factor receptor-bound protein 2 (Grb2) (12-15), TNFR-associated factor (TRAF) 3 (20), TRAF6 (19), DAP12 $(17,22)$, and Caspase recruitment domain-containing protein (Card) 9 (21).

Downstream of kinase 3 is itself activated by phosphorylation. A number of PTKs have been shown to phosphorylate Dok-3 and these include Abl (7), Src family tyrosine kinases such as Lyn (14) and Src and Tec family of kinases such as Bruton's tyrosine kinase (BTK) (20). In certain circumstances such as TLR4, 9 and CLR signaling, the phosphorylation of Dok-3 leads to its degradation and de-repression of immune receptor signaling (18-21).

The multiple kinases that can phosphorylate Dok-3 and the diverse classes of signaling molecules that can interact with Dok3 reveal the complexity of Dok-3 signaling and highlight its flexibility in orchestrating distinct signaling complexes to achieve specific cellular outcomes (Figure 3 and Table 1).

\section{ROLES OF Dok-3}

Over the past decades, Dok-3 has generally been implicated in the inhibition of immunoreceptor signaling pathways. However, we now know that this is not always the case. As the roles of Dok-3 in different cell types are starting to be unraveled, accumulating evidence suggests that Dok-3 can function, in a context-dependent manner, as both a positive and negative regulator of signaling pathways, in both immune as well as non-immune cells (Figure 3 and Table 1).

\section{B Cells}

Downstream of kinase 3 was discovered and cloned as a tyrosinephosphorylated protein which interacts with two inhibitory molecules, SHIP and Csk. SHIP, a $5^{\prime}$ inositol phosphatase implicated in the negative regulation of immunoreceptor signaling, is observed to bind to the PTB domain of Dok-3 via their tyrosine-phosphorylated residues, and this interaction is further facilitated by the association between their $\mathrm{SH} 2$ domain and the tyrosine-phosphorylated residues on Dok-3. On the other hand, Csk, a PTK involved in the negative regulation of Src family kinases, is able to contact the tyrosine-phosphorylated residues 
Phosphorylation

Acetylation
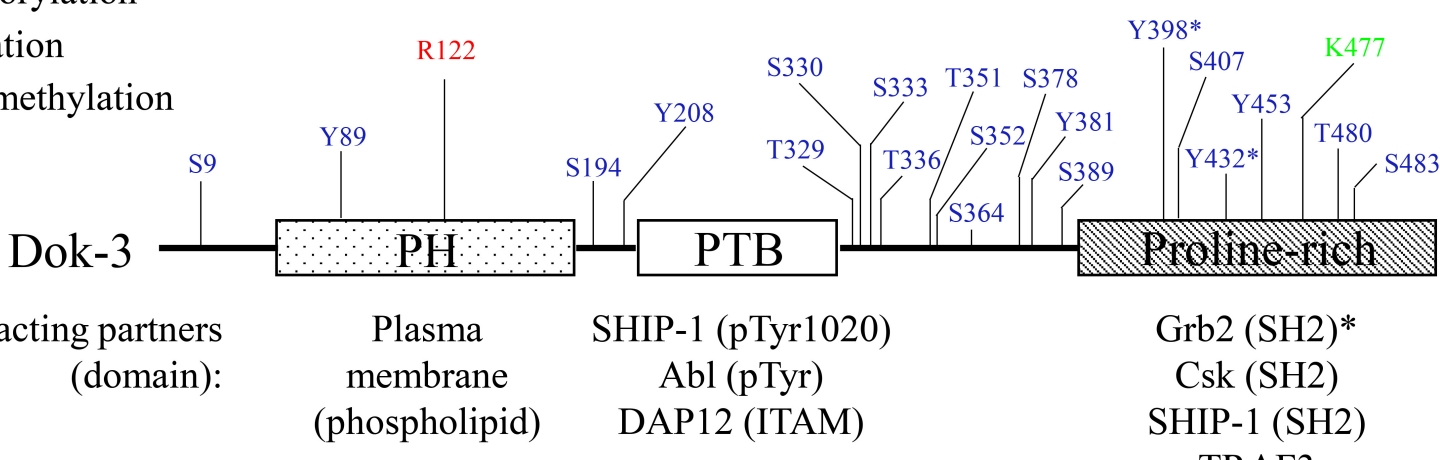

$\begin{array}{rcc}\text { Interacting partners } & \text { Plasma } & \text { SHIP-1 (pTyr1020) } \\ \text { (domain): } & \text { membrane } & \text { Abl (pTyr) } \\ & \text { (phospholipid) } & \text { DAP12 (ITAM) }\end{array}$

$\mathrm{Grb} 2(\mathrm{SH} 2)^{*}$
Csk (SH2)
SHIP-1 (SH2)

TRAF3

TBK1

FIGURE 1 | Schematic diagram of human Dok-3. Dok-3 has an amino-terminal pleckstrin homology (PH) and phosphotyrosine binding (PTB) domains, followed by a carboxy-terminal proline-rich region. Above, known phosphorylation, acetylation and methylation sites on Dok-3 mapped by mass spectrometry are indicated. Below, interaction partners of PH, PTB and proline-rich domains of Dok-3 are shown, together with their respective interacting domains/motifs/amino acid residues. The symbol "*” represents tyrosine residues on Dok-3 which bind specifically to SH2 domain of Grb2.

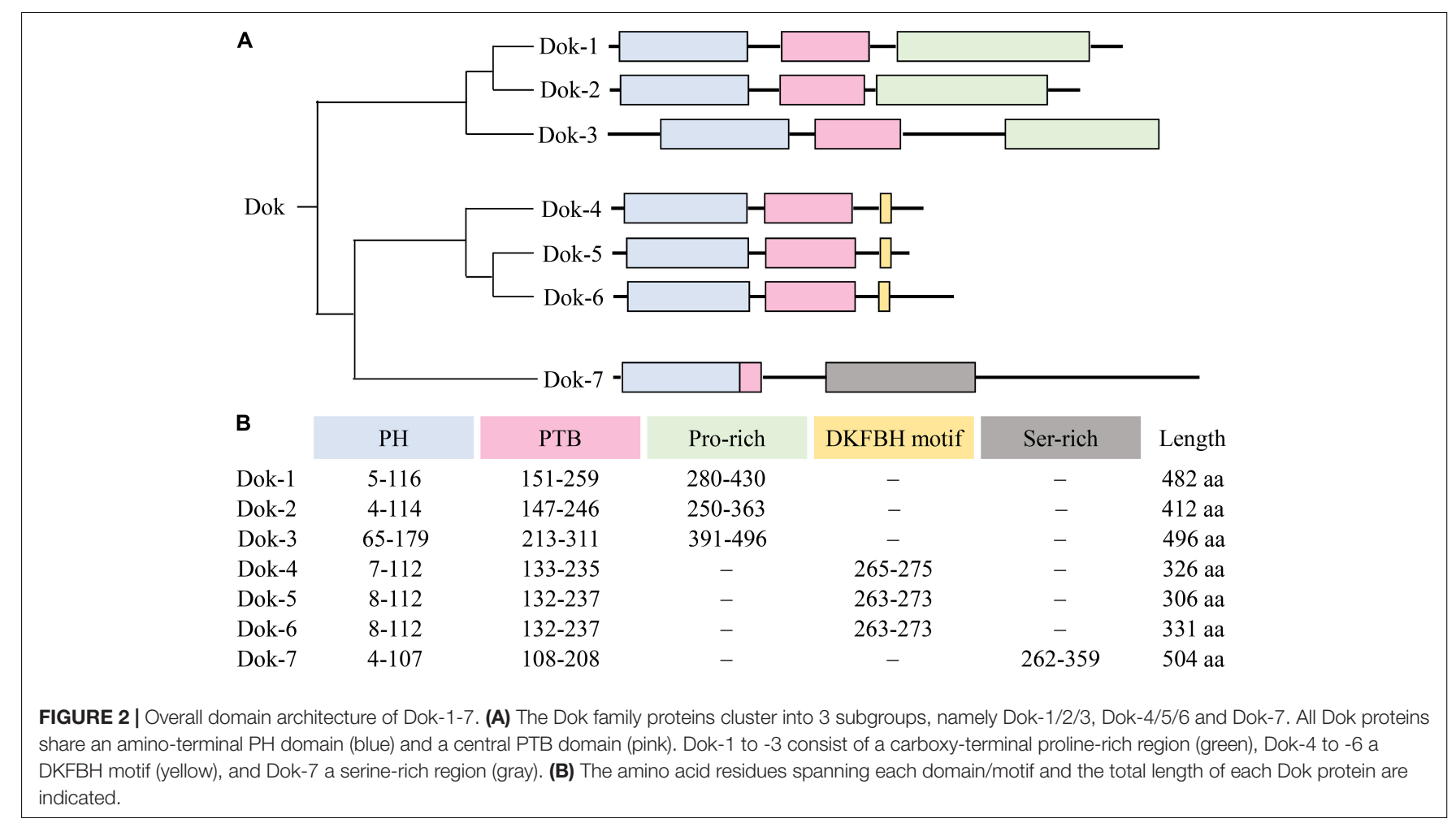

in the carboxy-terminal region of Dok-3 with their SH2 domain to mediate binding. Since Dok-3 has the tendency to interact with these two inhibitory molecules upon engagement of BCR, and overexpression of Dok-3 inhibited BCR-mediated nuclear factor of activated T-cells (NFAT) activation and Interleukin (IL)2 secretion in stimulated B cells, this study provided the first line of evidence that Dok-3 functions as a negative regulator of immunoreceptor signaling in B cells (8) (Figure 3A).

The inhibitory function of Dok-3 was further highlighted, when it was cloned by another group by virtue of its interaction with the oncoprotein Abl tyrosine kinase. The PTB domain of Dok-3 binds to the phosphotyrosine residues of $\mathrm{Abl}$ in a kinasedependent manner, and this is important for Abl-mediated tyrosine phosphorylation of Dok-3. Here, overexpression of Dok3 was found to inhibit Ras-MAP kinase pathway downstream of $\mathrm{Abl}$ and suppress its transforming ability in vivo (7) (Figure 3A).

Later studies further investigated the physiological relevance of Dok-3 in B cells, and showed its dispensability for early B cell development and the homeostasis of the peripheral $\mathrm{B}$ cell populations. Instead, Dok-3 was found to play an inhibitory 


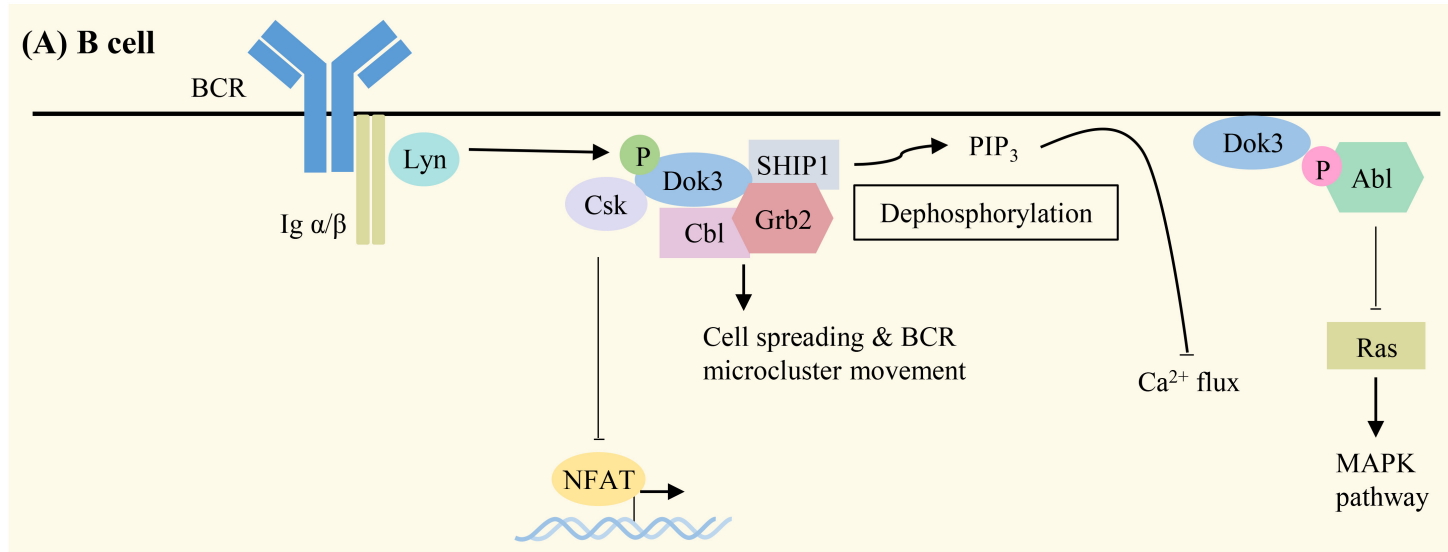

(B) Macrophage

(C) Neutrophil

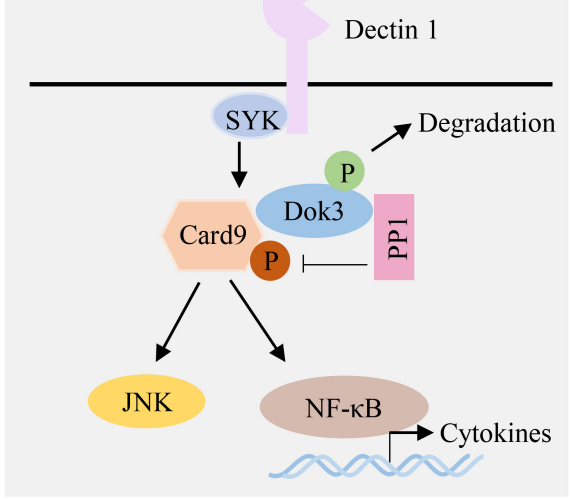

(D) Osteoclast precursor

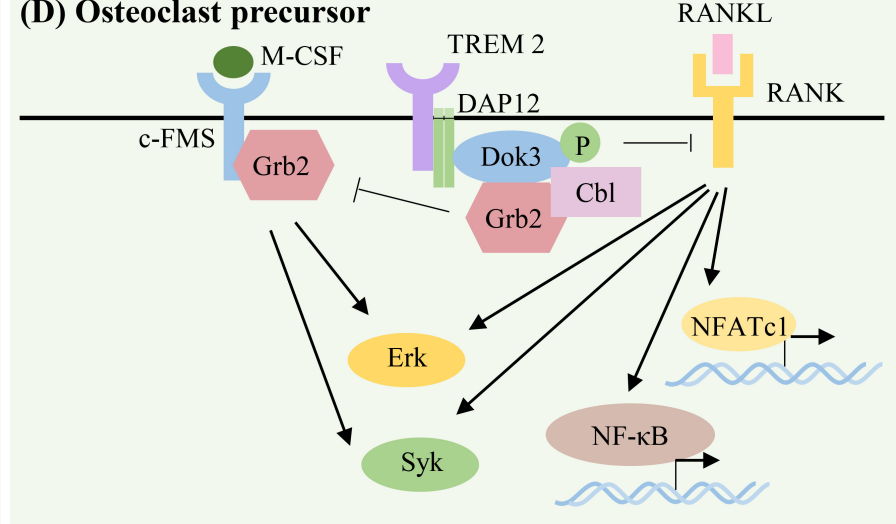

FIGURE 3 | Summary of Dok-3 signaling pathways in B cell, macrophage, neutrophil and osteoclast precursor. Dok-3 functions downstream of multiple receptors including B-cell receptor (BCR), Toll-like receptors (TLRs) and C-type lectin receptors (CLRs) to facilitate the nucleation of distinct signaling molecules and adapt them to regulate cellular functions in a spatially and temporally regulated manner. (A) Dok-3 limits BCR signaling by recruiting Grb2/SHIP1 to attenuate calcium flux, interacts with Csk to inhibit NFAT, or sequesters Abl to attenuate MAPK signaling. Dok-3 also associates with Grb2/Cbl to facilitate B cell spreading and transport of BCR microclusters for B cell activation to occur. (B) Dok-3 is phosphorylated upon low-dose or ubiquitinated and subsequently degraded upon high-dose LPS stimulation, thereby inhibiting TLR4 signaling in macrophages. Dok-3 also negatively regulates signaling downstream of TLR9. In contrast, Dok-3 is phosphorylated upon TLR3 activation leading to IRF3 induction and IFN $\beta$ production. (C) Dok-3 also participates in CLR signaling by recruiting PP1 to dephosphorylate Card9 for the attenuation of anti-fungal responses. (D) Dok-3 interacts with DAP12 to inhibit osteoclastogenesis. 
TABLE 1 | Roles of Dok-3 and their interacting partners in B cells, macrophages, neutrophils, osteoclasts, osteoblasts, and lung epithelial cells.

\begin{tabular}{|c|c|c|c|c|c|}
\hline Cell type & Activating/inhibitory & Function & Interacting partner & Mode of identification & References \\
\hline \multirow[t]{12}{*}{ B-cells } & Activating & Promotes plasma cell differentiation & - & - & $(16)$ \\
\hline & & Facilitates movement of BCR & Grb2 & Immuno-precipitation & $(15)$ \\
\hline & & microclusters for antigen gathering & $\mathrm{Cbl}$ & & \\
\hline & Inhibitory & Inhibits BCR signaling & SHIP & $\begin{array}{l}\text { Immuno-precipitation, in vitro binding } \\
\text { assay }\end{array}$ & (8) \\
\hline & & & Csk & Yeast two-hybrid, immuno-precipitation & \\
\hline & & & Lck & In vitro overexpression & \\
\hline & & & Fyn & & \\
\hline & & & Lyn & & \\
\hline & & $\begin{array}{l}\text { Inhibits Ras-MAPK pathway to } \\
\text { suppress transformation }\end{array}$ & $\mathrm{Abl}$ & Yeast two-hybrid, immuno-precipitation & $(7)$ \\
\hline & & Limits intracellular calcium signaling & Grb2 & Affinity Capture-MS & (13) \\
\hline & & & Lyn & Affinity Capture-Western & \\
\hline & & $\begin{array}{l}\text { Limits production of IgM and } \\
\text { proliferative capacity in response to } \\
\text { T-cell-independent type I and II } \\
\text { antigens }\end{array}$ & - & - & $(11)$ \\
\hline \multirow[t]{2}{*}{ Neutrophils } & Inhibitory & Inhibits Card9 signaling in response & Card9 & Immuno-precipitation & $(21)$ \\
\hline & & to fungal infection & PP1 & & \\
\hline \multirow[t]{9}{*}{ Macrophages } & Activating & Promotes IFN- $\beta$ production during & BTK & Immuno-precipitation & (20) \\
\hline & & viral infection & TRAF3 & & \\
\hline & & & TBK1 & & \\
\hline & Inhibitory & Inhibits TLR4 signaling & DAP12 & Immuno-precipitation & $(17,18)$ \\
\hline & & & Grb2 & & \\
\hline & & & SOS1 & & \\
\hline & & & Cbl-b & & \\
\hline & & $\begin{array}{l}\text { Negatively regulates IL- } 6 \text { and TNF- } \alpha \\
\text { production during TLR } 9 \text { signaling }\end{array}$ & TRAF6 & Immuno-precipitation & (19) \\
\hline & & $\begin{array}{l}\text { Inhibits malignant transformation } \\
\text { together with Dok-1 and Dok-2 }\end{array}$ & - & - & $(24)$ \\
\hline \multirow{3}{*}{$\begin{array}{l}\text { Osteoblasts and } \\
\text { osteoclasts }\end{array}$} & Activating & Promotes osteoblastogenesis & DAP12 & Immuno-precipitation & (22) \\
\hline & Inhibitory & Inhibits osteoclastogenesis & Grb2 & & \\
\hline & & & $\mathrm{Cbl}$ & & \\
\hline \multirow[t]{2}{*}{ Lung epithelial cells } & Inhibitory & $\begin{array}{l}\text { Inhibits lung tumorigenesis together } \\
\text { with Dok-1 and Dok-2 }\end{array}$ & - & - & (26) \\
\hline & & $\begin{array}{l}\text { Inhibits pulmonary inflammation } \\
\text { together with Dok-1 and Dok-2 }\end{array}$ & - & - & $(27)$ \\
\hline
\end{tabular}

role in BCR signaling to limit B cell production of $\operatorname{IgM}$ antibodies and their proliferative capacity in response to T-cellindependent type I and II antigens, but not to T-cell-dependent antigens. Downstream of kinase 3 knockout $\mathrm{B}$ cells exhibited greater induction of NF- $\mathrm{B}$, JNK and p38 signaling upon BCR stimulation, and their intracellular calcium signaling was also elevated at both low and high dose of stimulations. In the absence of Dok-3, the membrane recruitment of SHIP-1 was unaffected, but its phosphorylation and activation were compromised upon engagement of BCR. Hence, it is likely that Dok-3 exerts its inhibitory effect on BCR signaling through SHIP-1, although the exact mechanism as to how SHIP-1 activation is being controlled by Dok-3 was not further investigated (11).

It was demonstrated in separate studies, that calcium signaling downstream of BCR is attenuated by a Dok-3/Grb2/SHIP-1 module. In resting $\mathrm{B}$ cells, Dok-3 is localized to the plasma membrane via its $\mathrm{PH}$ domain, while the C-terminal SH3 domain of Grb2 interacts constitutively with the $\mathrm{SH} 3$ recognition motif ${ }^{1146}{ }^{\text {PPLPVK }}{ }^{1151}$ of SHIP-1 in the cytosol. Upon BCR stimulation, tyrosine phosphorylation of Dok-3 by Src family kinase Lyn promotes the recruitment of cytosolic Grb2/SHIP1 complex through the phospho-tyrosine (pTyr)-331/SH2 interaction between Dok-3 and Grb2, and PTB/pTyr-1020 within NPXY motif between Dok-3 and SHIP-1. As such, Dok-3 is able to regulate the subcellular localization of SHIP-1 and position it in the vicinity of plasma membrane for dephosphorylation of the lipid phosphoinositol 3,4,5-trisphosphate (PIP3). In this way, it is able to limit plasma membrane recruitment of BCR effector proteins and thus the efficiency of BCR signal transduction including intracellular calcium fluxes $(12,13)$ (Figure 3A).

The Dok-3/Grb2 module has also been shown to attenuate BCR signaling through the regulation of Lyn-dependent 
functions. Upon formation of a Dok-3/Grb2 complex at the plasma membrane, the C-terminal $\mathrm{SH} 3$ domain of Grb2 facilitates the translocation of the complex to BCR microsignalosomes. Within the microsignalosomes, the proximity of Dok-3/Grb2 complex to Lyn modulates the balance between their inhibitory and activating functions by inhibiting Lyn-dependent Syk phosphorylation and activation, while enhancing Lyn-dependent SHIP activation, thereby negatively regulating BCR signaling (14).

Although majority of the studies focused on the negative regulation of Dok-3 in BCR signaling, Dok-3 has also been reported to play a positive role during $B$ cell activation. Following antigen stimulation of BCR, Dok-3, together with Grb2 and Cbl, form a functional complex at the BCR microcluster to facilitate $B$ cell spreading and the efficient movement of microclusters for antigen gathering. Complex formation is initiated by Grb2 localization to the microcluster via their SH2 domain upon early B cell signaling, and this is required for the subsequent recruitment of Dok-3 and Cbl. Together, these three proteins promote the recruitment of microtubule motor dynein to mediate the transport of BCR microclusters along microtubule network for antigen gathering, as such allowing $\mathrm{B}$ cell activation to take place (15) (Figure 3A).

Apart from its role in controlling signaling pathways downstream of BCR in mature B cells, Dok-3 has also been shown to be required for plasma cell differentiation. Although Dok-3 knockout mice had an expansion of germinal center (GC) $\mathrm{B}$ and $\mathrm{T}$ follicular-helper $\left(\mathrm{T}_{\mathrm{fh}}\right)$ cells, the generation of antigenspecific plasma cells and their antibody responses were severely compromised. These Dok-3-deficient B cells were unable to sustain and upregulate the expression of programmed cell death 1 (PD-1) ligand 1 (PDL1) and PD-1 ligand 2 (PDL2) respectively, owing to enhanced calcium signaling due to the lack of negative regulation by Dok-3 as described above. Hence, Dok-3 plays a role in the antigen-driven phase of B-cell differentiation (16).

\section{Macrophages}

Downstream of kinase 3 has been implicated in the negative regulation of TLR4 signaling in bone marrow macrophages, as such preventing excessive inflammation and maintaining endotoxin tolerance upon continuous exposure to lipopolysaccharide (LPS). The PTB domain of Dok-3 binds to the immunoreceptor tyrosine-based activation motif (ITAM) of DAP12, an adaptor protein which couples to various cell surface immunoreceptors such as TLRs. Upon challenge with low-dose LPS, Dok-3 is tyrosine phosphorylated by Src family kinases in a DAP12-dependent manner, resulting in their translocation to the plasma membrane. Subsequently, tyrosine-phosphorylated Dok-3 associates with Grb2 and Son of sevenless homology 1 (SOS1) and sequesters Grb2 from SOS1 to inhibit downstream activation of the Ras-Erk pathway and the production of proinflammatory cytokines such as tumor necrosis factor (TNF) $\alpha$ (17). In HEK293 cells, pTyr-398 and pTyr-432 residues in the carboxy-terminal region of Dok-3 mediates its binding to the SH2 domain of Grb2 (23). Upon treatment with higher dosage of LPS, Dok-3 was ubiquitinated by the E3 ligase Cbl-b and degraded, triggering proteosomal degradation of SOS1 and further inhibiting Erk signaling (18). Hence, Dok-3 acts as an indispensable negative regulator of TLR4 signaling through two distinct mechanisms in macrophages. Likewise, Dok-3 is also shown to be a negative regulator of TLR9 signaling as its degradation by TRAF6-mediated ubiquitination is required for TLR 9-mediated IL-6 and TNF $\alpha$ production (19) (Figure 3B).

Contrary to its well-established role as a negative regulator downstream of immunoreceptors, Dok-3 has also been demonstrated to play a positive regulatory role in macrophages during interferon (IFN)- $\beta$ production in response to influenza virus infection or polyinosinic-polyribocytidylic acid [poly(I:C)] stimulation. Upon stimulation of TLR3, Dok-3 is tyrosine phosphorylated by BTK, and its carboxyl SH2-targeting domain forms a scaffold for the nucleation of TRAF3 and TBK1. As a result, TBK1 is activated by phosphorylation on Ser 172, possibly through autophosphorylation or by an unknown effector kinase, for the downstream induction of IFN regulatory factor (IRF) 3 and hence IFN- $\beta$ production (20) (Figure 3B).

The compensatory effect of Dok-3 for other closely related Dok family members, Dok-1 and Dok-2, has also been highlighted in the context of macrophages. Studies in mice showed that combined ablation of Dok-1, Dok-2, and Dok-3 promoted the development of histiocytic sarcoma, a malignant proliferation of tissue-resident macrophages. While macrophages lacking Dok-3 alone or Dok-1 and Dok-2 in combination did not show abnormalities, cells with combined loss of Dok-1, Dok-2, and Dok-3 demonstrated enhanced proliferation, suggesting that the Dok proteins mutually compensate for each other's function and cooperatively limit the proliferation of macrophages in response to macrophage colonystimulating factor (M-CSF) and granulocyte-macrophage colony-stimulating factor (GM-CSF). However, how ablation of Dok proteins can contribute to the malignant transformation of macrophages, leading to histiocytic sarcoma remains unknown (24). Specifically, it is still not known if Dok-3 plays a direct role downstream of growth factoror cytokine-receptors.

\section{Neutrophils}

High levels of Dok-3 mRNA have been detected in neutrophils as compared to other myeloid cell types. Here, Dok-3 is shown to negatively regulate the neutrophilic anti-fungal immune responses downstream of CLRs. During steady state, Dok-3 acts as an adaptor to bring Card9 and PP1 in close proximity, enabling the de-phosphorylation of Card9 on threonine residues, thereby suppressing Card9 activity. In this manner, it limits downstream signal transduction to NF- $\kappa \mathrm{B}$ and JNK, subsequently inhibiting anti-fungal effector functions such as phagocytosis, pro-inflammatory cytokines production and NETosis in neutrophils. Upon fungal infection, the tyrosine residues on Dok-3 are phosphorylated and Dok-3 is gradually degraded. This degradation leads to an enhancement of fungicidal properties in neutrophils. As a result, mice deficient in the negative regulator Dok-3 had a lower fungal burden in affected organs and were protected from lethal systemic infection with Candia albicans (21) (Figure 3C). 


\section{Osteoclasts and Osteoblasts}

Downstream of kinase 3 is required for the maintenance of normal bone homeostasis, and Dok-3 knockout mice were found to be osteoporotic, with increased osteoclastogenesis and reduced osteoblastogenesis. In the absence of Dok-3, osteoclast precursors showed increased proliferation in response to M-CSF (22) and increased cell-to-cell fusion of osteoclasts in response to receptor activator of nuclear factor- $\kappa \mathrm{B}$ ligand (RANKL) stimulation (25), resulting in an increased differentiation and resorption capacity. Mechanistically, M-CSF and RANKL signaling induced DAP12dependent phosphorylation of Dok-3, which subsequently forms an inhibitory multimeric complex with $\mathrm{Grb} 2$ and $\mathrm{Cbl}$; for the sequestration of Grb2 to limit downstream activation of Syk, NF- $\kappa$ B, Erk, and NFATc1 (Figure 3D). Excessive Erk activation in Dok-3 knockout cells enhances cyclin D1 expression, thereby promoting the proliferation of osteoclast precursors. In addition, Dok-3 was also found to regulate osteoclastogenesis through a DAP12-independent manner, since Dok-3 and DAP12 double knockout mice have normalized bone mass, unlike DAP12 knockout mice which maintained a high bone mass phenotype. On the other hand, Dok-3 promotes osteoblast differentiation and regulates their osteoprotegerin/RANKL ratio to provide another layer of regulation to osteoclast formation. Hence, Dok3 inhibits osteoclastogenesis and promotes osteoblastogenesis to regulate bone remodeling and prevent osteoporosis (22).

\section{Lungs}

Although the expression of Dok-3 is restricted mainly to hematopoietic cells, abundant amount of Dok- 3 was also detected in the non-lymphoid tissue lungs. Notably, mice deficient in Dok3 were found to develop lung adenocarcinoma, and the effect was more pronounced in mice with compound ablation of Dok-1, -2, and -3 , further demonstrating the overlapping and redundancies in function of the family of Dok proteins. Combined loss of Dok$1,-2$, and -3 enhanced phosphorylation and activation of Erk and Akt in lung epithelial cells, which promoted the expansion of bronchioalveolar stem cells (BASCs) and their differentiation into alveolar type II (AT2) cells, leading to neoplastic transformation of the lung. Indeed, these Dok genes were found to be expressed significantly in the BASC fraction of the lung, further supporting their role in preventing the malignant transformation of these progenitor cells. In particular, loss of Dok-3 was observed in 7\% of primary human lung adenocarcinoma samples from an arraybased comparative genomic hybridization dataset, and Dok-3 was also found to be decreased in lymph node metastases as compared to normal lung tissue, although their reduction was not as significant as that of Dok-2, a target of frequent copynumber loss in human lung cancer. Hence, Dok-1, -2 , and -3 act in concert as tumor suppressors of the lung, and their compound haploinsufficiency drives lung tumorigenesis in humans (26).

In addition, Dok-1, -2 , and -3 function cooperatively to prevent inflammation and maintain homeostasis in the lungs. While Dok-3 single knockout and Dok-1/2 double knockout mice were clinically well, combined ablation of these Dok proteins resulted in pulmonary inflammation, with increased infiltration of macrophages, eosinophils, neutrophils and lymphocytes in the

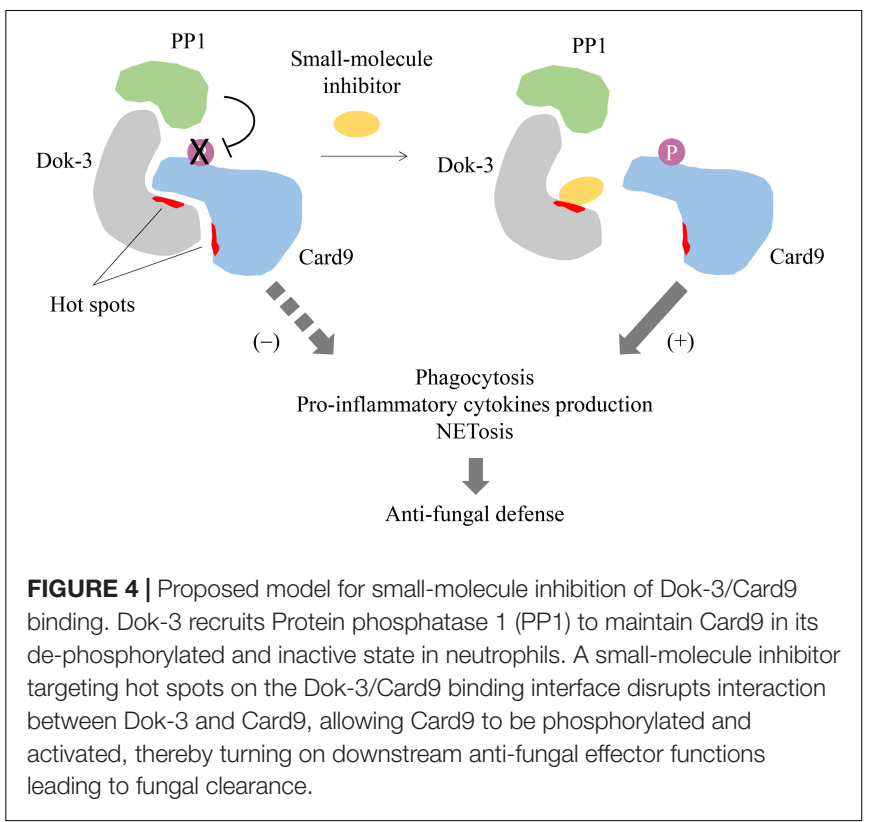

lungs, resulting in an asthma-like airway disorder. An increased production of Th2-type cytokines, including IL-4, -5, and -13, was observed in the bronchoalveolar lavage fluid (BALF) of the triple knockout mice, contributing to inflammation of the airway. Hence, Dok-1, -2 , and -3 act cooperatively to negatively regulate pulmonary inflammation (27).

\section{CONCLUSION AND FUTURE DIRECTIONS}

Adaptor proteins confer specificity in signaling events through their protein binding modules which dictate their binding partners, activity and subcellular localization, thereby regulating protein-protein interactions in a spatially and temporally regulated manner. Downstream of kinase 3 belongs to the Dok family of adaptor proteins, and it has some degree of homology, and as such overlapping functions, with other Dok members, in particular Dok-1 and -2. However, over the past years, emerging evidence suggests that Dok-3 plays a unique role in regulating cell signaling pathways via interaction with a distinct set of signal transducing molecules. Even though it was initially recognized as a negative regulator of immunoreceptor signaling, Dok-3 was later shown to possess both activating and inhibitory functions, in both lymphoid as well as non-lymphoid tissue. Hence, it is becoming clear that Dok-3 is a complex adaptor associated with a plethora of diverse cellular processes implicated in health and diseases such as cancer, bone homeostasis, immune suppression, as well as anti-viral and -fungal immunity. Since Dok-3 was also found to be expressed in organs like colon, reproductive tract and urinary bladder (10), it is interesting to speculate that it may play important roles in regulating other physiological functions in these organs. Moreover, since Dok-3 is known to interact with a variety of other signaling molecules, it remains to be determined if Dok-3 participates in the signal transduction of 
other immune receptors. Specifically, Dok-3 binds DAP12, which is itself an adaptor associated with numerous immune receptors such as killer immunoglobulin-like receptor (KIR), NKp44, NKG2C/CD94, and NKG2D (28-31). Hence, it is tempting to speculate that Dok-3 might play a role in natural killer (NK) cell functions as well. Thus, more studies need to be done in future for a greater appreciation and understanding of the roles of Dok-3 in signal transduction in various cell types and cell functions.

Given the importance of Dok-3 in the mechanistic regulation of cell signaling and its implication in human health and diseases, it will be exciting to see in near future the discovery of drugs which could block the specific interaction of Dok-3 with its various binding partners for prophylaxis or treatment of diseases. For instance, association between Dok-3 and Card9 is pivotal for suppressing Card9 signaling and hence restraining the fungicidal properties of neutrophils such as phagocytosis, pro-inflammatory cytokines release and NETs formation during fungal infection (21). By designing drugs which disrupt the specific interaction between these two molecules, we can potentially remove the brakes on anti-fungal immunity and enhance neutrophil-mediated clearance of fungal pathogens to combat candidaemia (Figure 4). Therefore, Dok-3 represents an important avenue for the development of human therapeutics against various diseases.

Even though adaptor proteins like Dok-3 are essentially viewed as "undruggable," mainly due to the lack of well-defined binding pockets which modulate protein-protein interactions, recent progress in drug discovery has proven that they are amenable to drug inhibition. High affinity regions termed "hot spots" which drive protein binding have been mapped onto protein-protein interfaces, and these hot spots are particularly adept at binding small-molecule or peptide inhibitors $(32,33)$. Such inhibitors of adaptors will have the advantage of higher efficacy and increased specificity, and as such reduced toxicity, as compared to conventional kinase inhibitors, which are often non-specific due to the highly conserved nature of the kinase domains (34). Hence, it will be feasible to design and develop inhibitors which disrupt Dok-3 binding with their interacting partners for therapeutic interventions. However, this pursuit is undoubtedly hindered by our limited understanding on the

\section{REFERENCES}

1. Yaffe MB. Phosphotyrosine-binding domains in signal transduction. Nat Rev Mol Cell Biol. (2002) 3:177-86. doi: 10.1038/nrm759

2. Mashima R, Hishida Y, Tezuka T, Yamanashi Y. The roles of Dok family adapters in immunoreceptor signaling. Immunol Rev. (2009) 232:273-85. doi: 10.1111/j.1600-065X.2009.00844.X

3. Yamanashi Y, Tamura T, Kanamori T, Yamane H, Nariuchi H, Yamamoto T, et al. Role of the rasGAP-associated docking protein p62(dok) in negative regulation of B cell receptor-mediated signaling. Genes Dev. (2000) 14:11-6.

4. Yasuda T, Shirakata M, Iwama A, Ishii A, Ebihara Y, Osawa M, et al. Role of Dok-1 and Dok-2 in myeloid homeostasis and suppression of leukemia. J Exp Med. (2004) 200:1681-7. doi: 10.1084/jem.20041247

5. Grimm J, Sachs M, Britsch S, Di Cesare S, Schwarz-Romond T, Alitalo K, et al. Novel p62dok family members, dok-4 and dok-5, are substrates of the c-Ret receptor tyrosine kinase and mediate neuronal differentiation. J Cell Biol. (2001) 154:345-54. doi: 10.1083/jcb.200102032 structure of Dok-3. Hot-spots on the binding interfaces between Dok-3 and its protein partners have to be defined to facilitate drug design. As such, it will be necessary for future studies to characterize the protein-protein interaction interfaces on Dok3 , typically through protein-based nuclear magnetic resonance (NMR) or X-ray crystallography, to assess its druggability and for the selection of appropriate drug screening and design strategies. Currently, therapeutic targeting of Dok-3 is still in its infancy, and a combination of biological, chemical and structural approaches is essential to propel its drug discovery forward.

Taken together, Dok-3 is a complex adaptor which forms an integral part of a highly diverse functional network within cells. Although we have gained rich knowledge of Dok-3 signaling and their interacting partners in recent years, its underlying expression and functions in other cell types, tissues and organs remain largely unexplored. With a clear understanding of its signaling mechanisms and physiological roles, there exists an opportunity for the development of Dok-3 inhibitors for clinical intervention of various diseases. Thus, we anticipate in the near future further progress in our understanding of Dok-3 in the field of signal transduction, and the emergence of small-molecule inhibitors of adaptor-binder interactions as a potential therapy to modulate human diseases.

\section{AUTHOR CONTRIBUTIONS}

JTL and JKHT wrote the manuscript. K-PL conceived the idea. $\mathrm{H}-\mathrm{HL}$ and K-PL edited and revised the manuscript. All authors contributed to the article and approved the submitted version.

\section{FUNDING}

This work is supported by the Singapore Ministry of Health's National Medical Research Council under its Open FundYoung Individual Research Grant (NMRC/OFYIRG/083/2018 to JTL) and Open Fund-Individual Research Grant (NMRC/OFIRG19may-0083 to K-PL, JTL, and H-HL), and an A*STAR core grant (to K-PL).
6. Crowder RJ, Enomoto H, Yang M, Johnson EM Jr., Milbrandt J. Dok-6, a Novel p62 Dok family member, promotes Ret-mediated neurite outgrowth. J Biol Chem. (2004) 279:42072-81. doi: 10.1074/jbc.M403726200

7. Cong F, Yuan B, Goff SP. Characterization of a novel member of the DOK family that binds and modulates Abl signaling. Mol Cell Biol. (1999) 19:831425. doi: $10.1128 / \mathrm{mcb} .19 .12 .8314$

8. Lemay S, Davidson D, Latour S, Veillette A. Dok-3, a novel adapter molecule involved in the negative regulation of immunoreceptor signaling. Mol Cell Biol. (2000) 20:2743-54. doi: 10.1128/mcb.20.8.2743-2754.2000

9. Favre C, Gerard A, Clauzier E, Pontarotti P, Olive D, Nunes JA. DOK4 and DOK5: new Dok-related genes expressed in human T cells. Genes Immun. (2003) 4:40-5. doi: 10.1038/sj.gene.6363891

10. Fagerberg L, Hallström BM, Oksvold P, Kampf C, Djureinovic D, Odeberg $\mathrm{J}$, et al. Analysis of the human tissue-specific expression by genomewide integration of transcriptomics and antibody-based proteomics. Mol Cell Proteom. (2014) 13:397-406. doi: 10.1074/mcp.M113.03 5600 
11. $\mathrm{Ng} \mathrm{CH}, \mathrm{Xu} \mathrm{S}, \mathrm{Lam} \mathrm{KP}$. Dok-3 plays a nonredundant role in negative regulation of B-cell activation. Blood. (2007) 110:259-66. doi: 10.1182/blood-2006-10055194

12. Manno B, Oellerich T, Schnyder T, Corso J, Losing M, Neumann K, et al. The Dok-3/Grb2 adaptor module promotes inducible association of the lipid phosphatase SHIP with the BCR in a coreceptor-independent manner. Eur J Immunol. (2016) 46:2520-30. doi: 10.1002/eji.201646431

13. Stork B, Neumann K, Goldbeck I, Alers S, Kahne T, Naumann M, et al. Subcellular localization of Grb2 by the adaptor protein Dok-3 restricts the intensity of Ca2+ signaling in B cells. EMBO J. (2007) 26:1140-9. doi: 10.1038/ sj.emboj. 7601557

14. Losing M, Goldbeck I, Manno B, Oellerich T, Schnyder T, Bohnenberger H, et al. The Dok-3/Grb2 protein signal module attenuates Lyn kinase-dependent activation of Syk kinase in B cell antigen receptor microclusters. J Biol Chem. (2013) 288:2303-13. doi: 10.1074/jbc.M112.406546

15. Schnyder T, Castello A, Feest C, Harwood NE, Oellerich T, Urlaub H, et al. $\mathrm{B}$ cell receptor-mediated antigen gathering requires ubiquitin ligase $\mathrm{Cbl}$ and adaptors Grb2 and Dok-3 to recruit dynein to the signaling microcluster. Immunity. (2011) 34:905-18. doi: 10.1016/j.immuni.2011.06.001

16. Ou X, Xu S, Li YF, Lam KP. Adaptor protein DOK3 promotes plasma cell differentiation by regulating the expression of programmed cell death 1 ligands. Proc Natl Acad Sci USA. (2014) 111:11431-6. doi: 10.1073/pnas. 1400539111

17. Peng Q, Long CL, Malhotra S, Humphrey MB. A physical interaction between the adaptor proteins DOK3 and DAP12 is required to inhibit lipopolysaccharide signaling in macrophages. Sci Signal. (2013) 6:ra72. doi: 10.1126/scisignal.2003801

18. Peng Q, O'Loughlin JL, Humphrey MB. DOK3 negatively regulates LPS responses and endotoxin tolerance. PLoS One. (2012) 7:e39967. doi: 10.1371/ journal.pone.0039967

19. Liu N, Tang B, Wei P, Sun W, Wang S, Peng Q. TRAF6-mediated degradation of DOK3 is required for production of IL-6 and TNF $\alpha$ in TLR9 signaling. Mol Immunol. (2015) 68(2 Pt C):699-705. doi: 10.1016/j.molimm.2015.10.021

20. Kim SS, Lee KG, Chin CS, Ng SK, Pereira NA, Xu S, et al. DOK3 is required for IFN-beta production by enabling TRAF3/TBK1 complex formation and IRF3 activation. J Immunol. (2014) 193:840-8. doi: 10.4049/jimmunol.1301601

21. Loh JT, Xu S, Huo JX, Kim SS, Wang Y, Lam KP. Dok3-protein phosphatase 1 interaction attenuates Card9 signaling and neutrophil-dependent antifungal immunity. J Clin Investigat. (2019) 129:2717-29. doi: 10.1172/jci126341

22. Cai X, Xing J, Long CL, Peng Q, Humphrey MB. DOK3 Modulates bone remodeling by negatively regulating osteoclastogenesis and positively regulating osteoblastogenesis. J Bone Mineral Res. (2017) 32:2207-18. doi: $10.1002 /$ jbmr.3205

23. Honma M, Higuchi O, Shirakata M, Yasuda T, Shibuya H, Iemura S, et al. Dok-3 sequesters Grb2 and inhibits the Ras-Erk pathway downstream of protein-tyrosine kinases. Genes Cells. (2006) 11:143-51. doi: 10.1111/j.13652443.2006.00926.x

24. Mashima R, Honda K, Yang Y, Morita Y, Inoue A, Arimura S, et al. Mice lacking Dok-1, Dok-2, and Dok-3 succumb to aggressive histiocytic sarcoma. Lab Invest. (2010) 90:1357-64. doi: 10.1038/labinvest.2010.121

25. Kajikawa S, Taguchi Y, Hayata T, Ezura Y, Ueta R, Arimura S, et al. Dok-3 and Dok-1/-2 adaptors play distinctive roles in cell fusion and proliferation during osteoclastogenesis and cooperatively protect mice from osteopenia. Biochem Biophys Res Commun. (2018) 498:967-74. doi: 10.1016/j.bbrc.2018.03.090

26. Berger AH, Niki M, Morotti A, Taylor BS, Socci ND, Viale A, et al. Identification of DOK genes as lung tumor suppressors. Nat Genet. (2010) 42:216-23. doi: 10.1038/ng.527

27. Mashima R, Arimura S, Kajikawa S, Oda H, Nakae S, Yamanashi Y. Dok adaptors play anti-inflammatory roles in pulmonary homeostasis. Genes Cells. (2013) 18:56-65. doi: 10.1111/gtc.12016

28. Lanier LL. DAP10- and DAP12-associated receptors in innate immunity. Immunol Rev. (2009) 227:150-60. doi: 10.1111/j.1600-065X.2008.00720.x

29. Lanier LL, Corliss B, Wu J, Phillips JH. Association of DAP12 with activating CD94/NKG2C NK cell receptors. Immunity. (1998) 8:693-701. doi: 10.1016/ s1074-7613(00)80574-9

30. Campbell KS, Yusa S, Kikuchi-Maki A, Catina TL. NKp44 triggers NK cell activation through DAP12 association that is not influenced by a putative cytoplasmic inhibitory sequence. J Immunol. (2004) 172:899-906. doi: 10. 4049/jimmunol.172.2.899

31. Wensveen FM, Jelenčić V, Polić B. NKG2D: a master regulator of immune cell responsiveness. Front Immunol. (2018) 9:441. doi: 10.3389/fimmu.2018.00441

32. Scott DE, Bayly AR, Abell C, Skidmore J. Small molecules, big targets: drug discovery faces the protein-protein interaction challenge. Nat Rev Drug Discov. (2016) 15:533-50. doi: 10.1038/nrd.2016.29

33. Arkin MR, Wells JA. Small-molecule inhibitors of protein-protein interactions: progressing towards the dream. Nat Rev Drug Discov. (2004) 3:301-17. doi: $10.1038 /$ nrd1343

34. Luo LY, Hahn WC. Oncogenic signaling adaptor proteins. J Genet Genomics Chuan Xue Bao. (2015) 42:521-9. doi: 10.1016/j.jgg.2015.09.001

Conflict of Interest: The authors declare that the research was conducted in the absence of any commercial or financial relationships that could be construed as a potential conflict of interest.

Copyright (c) 2020 Loh, Teo, Lim and Lam. This is an open-access article distributed under the terms of the Creative Commons Attribution License (CC BY). The use, distribution or reproduction in other forums is permitted, provided the original author(s) and the copyright owner(s) are credited and that the original publication in this journal is cited, in accordance with accepted academic practice. No use, distribution or reproduction is permitted which does not comply with these terms. 